\title{
Nanocomposites Research in Tongji University: Introduction of Prof. Hongbo Gu
}

Henry A. Colorado, ${ }^{1 *}$ Qiang Gao, ${ }^{2 *}$ Subramania Angaiah, ${ }^{3^{*}}$ Zhe Wang $,{ }^{4 *} \mathrm{Na} \mathrm{Lu},{ }^{5^{*}}$ Ying Li, ${ }^{6^{*}}$ Jong Eun Ryu, ${ }^{7^{*}}$ Brian J. Edwards ${ }^{8^{*}}$ and David P. Young ${ }^{* *}$

Accepted 16 November 2018

DOI: $10.30919 / \mathrm{es} 8 \mathrm{~d} 624$

\section{Hongbo Gu}

(Email: hongbogu2014@tongji.edu.cn)

As one of the top 10 universities in China, Tongji University is the leading research institution in the area of nanocomposites. Dr. Hongbo Gu, an Associate Professor in the School of Chemical Science and Engineering at Tongji University, is leading the nanocomposites and electronic devices research in the university. Dr. Gu received her Bachelor's (2008) and Ph.D. degree (2014) in Chemical Engineering and Technology at the Harbin Institute of Technology (HIT), China. During her graduate study, she worked as a joint Chemical Engineering Ph.D. student at Lamar University, TX, USA, while being sponsored by the China Scholarship Council (CSC). Dr. Gu joined Tongji University in 2014 as an assistant professor and was tenured in 2018. $\mathrm{Dr}$. $\mathrm{Gu}$ has worked for more than ten years in the design and development of multifunctional advanced polymer nanocomposites, especially in the area of magnetic and conductive materials for use in electronic devices and for environmental remediation.

While Dr. Gu's research has contributed broadly to the field of nanocomposites, she has devoted a significant amount of her research to the following three thrust areas:

(1) Discovery of room-temperature giant magnetoresistance (GMR) in polyaniline (PANI) and its nanocomposites, ${ }^{1-3}$ especially in those that are non-magnetic (up to $95.5 \%$ GMR). ${ }^{4}$ Generally, PANI, as one of the few organic conducting polymers, possesses a temperaturedependent GMR effect, in which the GMR phenomenon is often attained at low temperature. Dr. Gu's group has studied the GMR

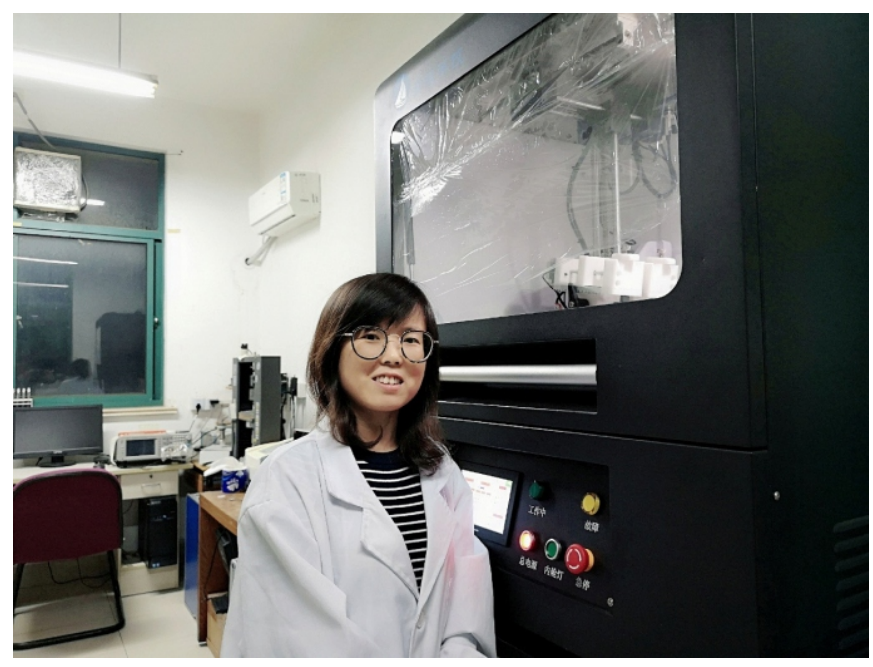

Dr. Hongbo Gu standing near electrospinning equipment in Tongji University - a top 10 university in China.

effect in the PANI system for a long-term and found that the synthesis method, dopant, doping degree, crystallinity, and the type and loading of nanofillers significantly influence the GMR values in the PANI and its nanocomposites. By adjusting these parameters, Dr. Gu's group has reported on the PANI systems with high GMR values

\footnotetext{
CCComposites Laboratory, Universidad de Antioquia (UdeA), Calle 70 No. 52-21, Medellin, Colombia

${ }^{2}$ Max Planck Institute for Chemical Energy Conversion, Stiftstrasse 34-36, 45470 Mülheim an der Ruhr, Germany

${ }^{3}$ Centre for Nanoscience and Technology, Pondicherry University, Puducherry 605 014, India

${ }^{4}$ Chemistry Department, Xavier University of Louisiana, New Orleans, LA, USA

${ }^{5}$ Lyles School of Civil Engineering, School of Materials Engineering, Birck

Nanotechnology Center, Purdue University, West Lafayette, 47906, USA

${ }^{6}$ Department of Mechanical Engineering, Texas A\&M University, College Station, TX 77843, USA

${ }^{7}$ Department of Mechanical Engineering and Integrated Nanosystems Development Institute, Indiana University-Purdue University Indianapolis, Indianapolis, IN 46202, USA

${ }^{8}$ Materials Research and Innovation Laboratory (MRAIL), Department of Chemical and Biomolecular Engineering, University of Tennessee, Knoxville, Tennessee 37996, USA

${ }^{9}$ Department of Physics and Astronomy, Louisiana State University, Baton Rouge, LA 70803, USA

*E-mail: henry.colorado@udea.edu.co; qiang.gao@cec.mpg.de; asubramania.nst@pondiuni.edu.in; zwang@xula.edu; luna@purdue.edu; yingli@tamu.edu; jryu@ncsu.edu; bedward1@utk.edu; dyoung@phys.lsu.edu
} 

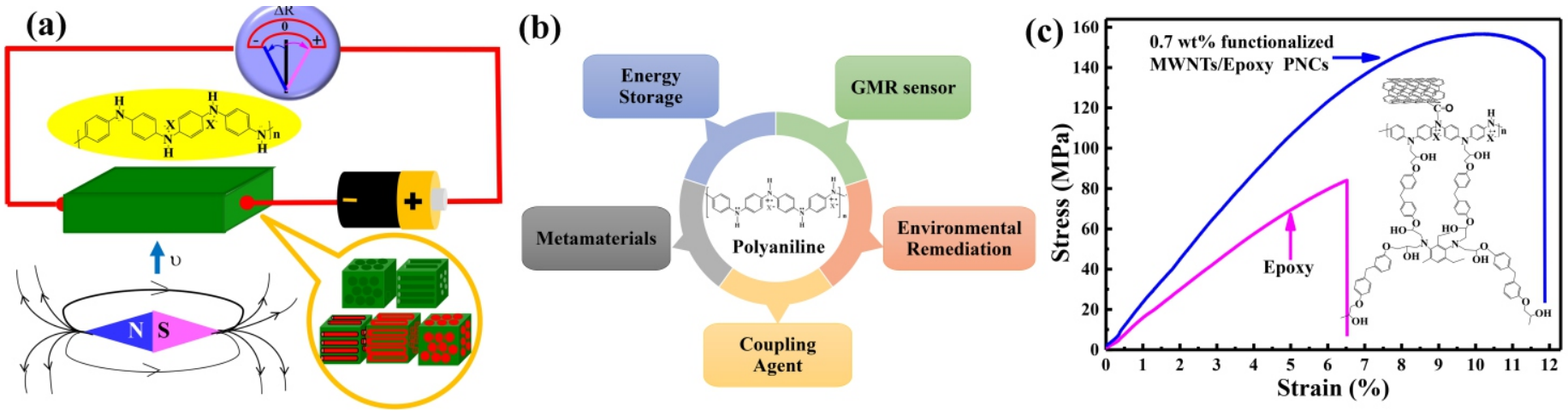

Fig. 1 (a) Illustration of polyaniline and its nanocomposites with the GMR effect; (b) new functions of polyaniline; (c) enhanced tensile strength for functionalized multiwalled carbon nanotubes/epoxy polymer nanocomposites (PNCs) with polyaniline as a coupling agent.

at room temperature. Their goal is to prepare the PANI system with larger GMR signals and higher GMR sensitivity at low magnetic field, as well as the potential applications of conducting polymers for GMR sensors in large-scale practical applications, Fig. 1a.

(2) Development of new applications of PANI materials, such as room temperature GMR sensors, ${ }^{5-7}$ coupling agent for epoxy and nanofillers, ${ }^{8-14}$ and metamaterials with negative permittivity, Fig $1 b^{2,15-17}$ PANI has been one of the most thoroughly investigated conducting polymers over the past years for applications of supercapacitors, gas separation membranes, electrochromic materials, and anti-corrosion materials. It's essential to discover new applications and further broaden the technical scope of PANI. By utilizing advanced structure design, Dr. Gu's group has developed new applications of PANI, such as room temperature GMR sensors, a coupling agent for epoxy and nanofillers (the tensile strength of epoxy can be increased by $85 \%$ after introducing the PANI on the surface of multi-walled carbon nanotubes, Fig. $1 \mathrm{c}^{12}$ ), and metamaterials with negative permittivity (the negative permittivity of PANI can be tuned by adding different morphologies and loadings of nanofillers.)

(3) And finally, environmental remediation using carbonprotected magnetic nanocomposites. ${ }^{18-20}$ Normally, the presence of transition metals such as $\mathrm{Fe}, \mathrm{Co}$, and $\mathrm{Ni}$ nanoparticles with various magnetic properties can help recycle adsorbents after the treatment of polluted water. However, one challenge to overcome is the easy oxidation/corrosion of the magnetic nanoparticles, which leads to a diminished magnetization. To maintain a long-term stability of the magnetic nanoparticles, Dr. Gu's group has fabricated a noble carbon layer to protect them from harsh environments, such as acids. Furthermore, the carbon-protected materials with a large specific surface area can also improve pollutant-adsorption performance of the final nanocomposites. Based on this idea, Dr. Gu's group has exploited several different magnetic carbon nanocomposites for remediation of heavy metal ions and organic pollutants.

At present, Dr. Gu has published more than 70 scientific peerreviewed papers that have been well recognized by the scientific community (3600+ citations) with an $h$-index of 32 .

In addition, she actively serves as a deputy editor of $E S$ Materials \& Manufacturing and is an executive editorial board member of ES Energy \& Environment. She is an advisory board member of Engineered Science and Advanced Composites and Hybrid Materials. She was awarded a participation fellowship from the 2012 NSF-CMMI research and innovation conference.

\section{References:}

1. H. Gu, X. Zhang, H. Wei, Y. Huang, S. Wei and Z. Guo, Chem. Soc. Rev., 2013, 42, 5907-5943.

2. H. Gu, Y. Huang, X. Zhang, Q. Wang, J. Zhu, L. Shao, N. Haldolaarachchige, D. P. Young, S. Wei and Z. Guo, Polymer, 2012, 53, 801-809.

3. H. Gu, J. Guo, X. Yan, H. Wei, X. Zhang, J. Liu, Y. Huang, S. Wei and Z. Guo, Polymer, 2014, 55, 4405-4419.

4. H. Gu, J. Guo, X. Zhang, Q. He, Y. Huang, H. A. Colorado, N. S. Haldolaarachchige, H. L. Xin, D. P. Young, S. Wei and Z. Guo, J. Phys. Chem. C, 2013, 117, 6426-6436.

5. H. Gu, H. Zhang, J. Lin, Q. Shao, D. P. Young, L. Sun, T. D. Shen and Z. Guo, Polymer, 2018, 143, 324-330.

6. H. Gu, J. Guo, H. We, Y. Huang, C. Zhao, Y. Li, Q. Wu, N. Haldolaarachchige, D. P. Young, S. Wei and Z. Guo, Phys. Chem. Chem. Phys., 2013, 15, 10866-10875.

7. H. Gu, J. Guo, R. Sadu, Y. Huang, N. Haldolaarachchige, D. Chen, D. P. Young, S. Wei and Z. Guo, Appl. Phys. Lett., 2013, 102, 212403.

8. H. Gu, C. Ma, J. Gu, J. Guo, X. Yan, J. Huang, Q. Zhang and Z. Guo, J. Mater. Chem. C, 2016, 4, 5890-5906.

9. H. Gu, H. Zhang, C. Ma, S. Lyu, F. Yao, C. Liang, X. Yang, J. Guo, Z. Guo and J. Gu, J. Phys. Chem. C, 2017, 121, 1326513273.

10. H. Gu, J. Guo, H. Wei, S. Guo, J. Liu, Y. Huang, A. Khan Mojammel, X. Wang, P. Young David, S. Wei and Z. Guo, Adv. Mater., 2015, 27, 6277-6282.

11. H. Gu, C. Ma, C. Liang, X. Meng, J. Gu and Z. Guo, J. Mater. Chem. C, 2017, 5, 4275-4285.

12. H. Gu, S. Tadakamalla, X. Zhang, Y.-D. Huang, Y. Jiang, H. A. Colorado, Z. Luo, S. Wei and Z. Guo, J. Mater. Chem. C, 2013, 1, 729-743.

13. H. Gu, S. Tadakamalla, Y. Huang, H. A. Colorado, Z. Luo, N. Haldolaarachchige, D. P. Young, S. Wei and Z. Guo, ACS Appl. Mater. Interfaces, 2012, 4, 5613-5624.

14. H. Gu, J. Guo, Q. He, S. Tadakamalla, X. Zhang, X. Yan, Y. Huang, H. A. Colorado, S. Wei and Z. Guo, Ind. Eng. Chem. Res., 2013, 52, 7718-7728.

15. H. Gu, J. Guo, Q. He, Y. Jiang, Y. Huang, N. Haldolaarachige, Z. Luo, D. P. Young, S. Wei and Z. Guo, Nanoscale, 2014, 6, 181189.

16. H. Gu, J. Guo, M. A. Khan, D. P. Young, T. D. Shen, S. Wei and Z. Guo, Phys. Chem. Chem. Phys., 2016, 18, 19536-19543. 
17. H. Gu, J. Guo, S. Wei and Z. Guo, J. Appl. Polym. Sci., 2013, 130, 2238-2244.

18. X. Xu, H. Zhang, C. Ma, H. Gu, H. Lou, S. Lyu, C. Liang, J. Kong and J. Gu, J. Hazard. Mater., 2018, 353, 166-172.
19. B. Xiang, D. Ling, H. Lou and H. Gu, J. Hazard. Mater, 2017, 325, 178-188.

20. H. Gu, H. Lou, J. Tian, S. Liu and Y. Tang, J. Mater. Chem. A, 2016, 4, 10174-10185 